\title{
Whole exome sequencing of an asbestos- induced wild-type murine model of malignant mesothelioma
}

Sophie Sneddon ${ }^{1}$, Ann-Marie Patch², lan M. Dick', Stephen Kazakoff², John V. Pearson², Nicola Waddell², Richard J. N. Allcock ${ }^{3,4}$, Robert A. Holt ${ }^{5}$, Bruce W. S. Robinson ${ }^{1,6}$ and Jenette Creaney ${ }^{1^{*}}$

\begin{abstract}
Background: Malignant mesothelioma (MM) is an aggressive cancer of the pleural and peritoneal cavities caused by exposure to asbestos. Asbestos-induced mesotheliomas in wild-type mice have been used extensively as a preclinical model because they are phenotypically identical to their human counterpart. However, it is not known if the genetic lesions in these mice tumours are similar to in the human disease, a prerequisite for any new preclinical studies that target genetic abnormalities.
\end{abstract}

Methods: We performed whole exome sequencing of fifteen asbestos-induced murine MM tumour cell lines from $\mathrm{BALB} / \mathrm{C}, \mathrm{CBA}$ and $\mathrm{C} 57 \mathrm{BL} / 6$ mouse strains and compared the somatic mutations and copy number variations with those recurrently reported in human $\mathrm{MM}$. We then catalogued and characterised the mutational landscape of the wild-type murine MM tumours. Quantitative RT-PCR was used to interrogate the expression of key MM genes of interest in the mRNA.

Results: Consistent with human MM tumours, we identified homozygous loss of the tumour suppressor Cdkn2a in 14/15 tumours. One tumour retained the first exon of both of the p16INK4a and p19ARF isoforms though this tumour also contained genetic amplification of Myc resulting in increased expression of the c-Myc proto-oncogene in the mRNA. There were no chromosomal losses in either the Bap1 or Nf2 regions. One tumour harbored homozygous loss of Trp53 in the DNA. Mutation rates were similar in tumours generated in the CBA and C57BL/6 strains when compared to human MM. Interestingly, all BALB/c tumour lines displayed high mutational loads, consistent with the known mutator phenotype of the host strain. The Wnt, MAPK and Jak-STAT signaling pathways were found to be the most commonly affected biological pathways. Mutations and copy number deletions also occurred in the Hedgehog and Hippo pathways.

Conclusions: These data suggest that in the wild-type murine model asbestos causes mesotheliomas in a similar way to in human MM. This further supports the notion that the murine model of MM represents a genuine homologue of the human disease, something uncommon in cancer, and is thus a valuable tool to provide insight into MM tumour development and to aide the search for novel therapeutic strategies.

Keywords: Mesothelioma, Asbestos, Exome sequencing, Cdkn2a, Mouse model, Wild-type

\footnotetext{
* Correspondence: jenette.creaney@uwa.edu.au

${ }^{1}$ National Centre for Asbestos Related Disease, School of Medicine and

Pharmacology, QEIl Medical Centre, University of Western Australia, QQ Block,

6 Verdun Street, Nedlands, WA 6009, Australia

Full list of author information is available at the end of the article
} 


\section{Background}

Malignant mesothelioma (MM) is an aggressive cancer caused by DNA damage in the mesothelial cells of the pleural and peritoneal cavities that principally results following asbestos exposure [1-3]. There is a long latency between asbestos exposure and tumour development with periods of $30-50$ years frequently reported. MM is almost universally fatal and median survival after diagnosis is short (9-12 months) [1, 2].

Asbestos is one of only a few carcinogens, along with cigarette smoke, UV light and several others, that can be readily identified in individuals. Importantly, asbestos induces mesotheliomas in mice which are almost identical to their human counterpart in terms of pathology, immunology and clinical behavior, which is rare in mouse modeling of cancer $[4,5]$. Although this has made the mouse model a useful preclinical tool for studying mesothelioma treatments, until now there has been no detailed study of the genetic lesions associated with mouse mesothelioma to determine if such lesions also parallel the human disease. Detailed studies of the genetic lesions of human MM have only recently been published. On average, human MM tumours contain less than 1 mutation per million bases, which is lower than other malignancies associated with external carcinogens such as lung cancer and melanoma [6-8]. A variety of somatic copy number variations ( $\mathrm{CNVs}$ ) and single nucleotide variations (SNVs) have been recurrently reported in several genes. Loss of $C D K N 2 A$, located at 9p21, is reported in more than $70 \%$ of cases; loss of NF2, located at $22 \mathrm{q}$, is reported in around $40 \%$ of cases; and mutations in $B A P 1$, located at 3p21.1 are reported in $40-60 \%$ of cases [9-13]. Recent large scale genomic analyses have also found recurrent, low frequency mutations in a number of additional genes including LATS2 (22\%), CUL1 (9\%), TP53 (8\%) and SETD2 (8\%) [7, 8, 14]. Shared somatic mutations between MM tumours are rare, however several biological pathways are often reported as being dysregulated in human MM, including the Wnt, Hedgehog, Notch, Ras, p53, MAPK, mTOR and Hippo signaling pathways $[7,15,16]$.

Murine models of $\mathrm{MM}$ are an invaluable tool for the preclinical evaluation of disease pathogenesis and for developing novel treatment strategies [17]. Recent studies have utilized mouse xenograft models and several genetically engineered mouse models to recapitulate the common mutations seen in human MM, such as $N f 2, C d k n 2 a$ and Bap1 knockout models [18-20]. However, such models only enable the study of MM in the context of the effect of the knocked out gene of interest. Our well established asbestos-induced wild-type murine MM model has the potential to offer useful molecular insights on the natural initiation and progression of MM in response to asbestos exposure, providing the opportunity for better understanding of pathogenesis, development of novel treatments and biomarker/signature discovery. Detailed characterisation of the genomic lesions underpinning the wild-type murine MM model have, as in other cancers, lagged behind the relevant human studies. This model has been extensively characterised at the phenotypic and morphological level on the BALB/c background [5]. Gene expression has previously been characterised in the C57BL/6 strain [21] and arraycomparative genomic hybridisation (aCGH) studies have identified lesions in FVB/N mice [22] however little is known about the mutational landscape of these wild-type tumours. We therefore undertook to characterize the somatic DNA lesions that underlie murine $\mathrm{MM}$ and characterise the mutational landscape using whole exome sequencing of fifteen independent murine MM tumour cell lines derived from three murine strains, comparing the mutations identified with those most often found in human MM.

\section{Methods \\ Sample collection and DNA extraction}

Fifteen murine MM cell lines were previously established from ascites generated following intra-peritoneal crocidolite asbestos injection into BALB/c $(n=4)$ [4], CBA $(n=5)[4]$ and C57BL/6 $(n=6)$ mice $[23,24]$ (Table 1$)$. Established cell lines (median passage 14, range 8-31) were used in this study and all cell lines were confirmed

Table 1 Establishment and growth rate information of wildtype asbestos-induced murine MM tumour cell lines

\begin{tabular}{lllll}
\hline Strain & $\begin{array}{l}\text { Cell line } \\
\text { name }\end{array}$ & $\begin{array}{l}\text { Year } \\
\text { established }\end{array}$ & $\begin{array}{l}\text { In vivo growth } \\
\text { rate }^{\text {a }}\end{array}$ & $\begin{array}{l}\text { Passage } \\
\text { sequenced }\end{array}$ \\
\hline BALB/C & AB1 & 1992 & fast & 18 \\
& AB12 & 1992 & slow & 11 \\
& AB13 & 1992 & slow & 14 \\
& AB22 & 1992 & slow & 8 \\
CBA & AC16 & 1992 & slow & 24 \\
& AC24 & 1992 & Undetermined & 31 \\
& AC28 & 1992 & slow & 13 \\
& AC29 & 1992 & fast & 31 \\
& AC31 & 1992 & fast & 8 \\
C57BL/6 & AE3 & 2001 & nil & 11 \\
& AE16 & 2001 & nil & 14 \\
& AE17 & 2001 & fast & 13 \\
& AE19 & 2001 & slow & 17 \\
& BM109 & 2004 & slow & 14 \\
BM163 & 2005 & slow & 20 \\
\hline
\end{tabular}

Relative time taken for a $100 \mathrm{~mm}^{2}$ subcutaneous tumour to form following an inoculation of $5 \times 10^{5}$ cells in the flank of syngeneic mice. Growth rate slow $>80$ days; Fast $<40$ days 
free of Mycoplasma spp. infection by polymerase chain reaction. Cells were cultured as previously described [25]. Control DNA and RNA samples were obtained from current generation mice sourced from the Animal Resource Centre (Murdoch, WA, Australia) for each mouse strain in accordance with protocols approved by the University of Western Australia Animal Ethics Committee. DNA was extracted using standard isopropanol purification. RNA was extracted using an RNeasy Mini Kit (QIAGEN, Chadstone, Vic, Australia) and cDNA prepared using an OmniScript Reverse Transcription kit (QIAGEN) with oligo dT primers (Promega, Alexandria, NSW, Australia) following the manufacturer's instructions.

\section{Library preparation and exome sequencing}

Genomic DNA was fragmented using an S2 sonicator (Covaris, USA). Whole genome libraries were constructed using a NEBNext Ultra DNA Library kit (New England Biosciences, Ipswich, MA) with local modifications (specifically Ion Torrent sequencing adaptors were added) and the exome was enriched using a SureSelectXT Mouse All Exon kit (Agilent, Mulgrave, Vic, Australia) that targets $49.6 \mathrm{Mb}$ of the murine genome (221,784 exons across 24,306 coding genes). Sequencing was performed using a Proton sequencer (Ion Torrent, Thermofisher Scientific) system according to manufacturer's instructions at the Lotterywest State Biomedical Facility Genomics (Nedlands, WA, Australia). Two samples were pooled and sequenced on a P1 chip for 520 flows to an average depth of $42 \times$, which resulted in $80 \%$ of the targeted regions being covered with at least an average depth of $13.2 \times$ (See Additional file 1).

\section{Data processing and variant detection}

Sequence reads were trimmed using Cutadapt (version 1.9) [26], aligned to GRCh $38 / \mathrm{mm} 10$ with BWA-MEM (version 0.7.13-r1126), duplicates marked with Picard (version 1.141, http://broadinstitute.github.io/picard/) and coordinates sorted using Samtools (version 1.3) [27]. Single nucleotide substitution variants were detected using a dual calling strategy using qSNP [28] and the Haplotype caller module of GATK [29]. Short indels of $\leq 50 \mathrm{bp}$, were called with the Haplotype. Initial read quality filtering for all variants detected included: a minimum of 35 bases in the CIGAR string indicated a match, 3 or fewer mismatches in the sequencing MD field, and a mapping quality greater than 10 . High confidence variants were selected based on passing further variant characteristic filtering requirements and were used in all downstream analyses. These filtering requirements were: a minimum coverage of 8 reads in the control data and 12 reads in the tumour data and a maximum of 1000 reads; at least 5 variant supporting reads present where the variant was not within the first or last 5 bases; at least 4 of the 5 reads with individual start positions; the variant was identified in reads of both sequencing directions; the variant was not less than 5 base pairs from a mono-nucleotide run of 7 or more bases in length; and for somatic variants less than $3 \%$ variant evidence identified in the control sample. In addition, for indels a minimum of 4 strong variant supporting reads containing a maximum of 3 variant events (including the indel of interest and any other non-reference occurrence) were required. Indels that were located immediately adjacent to homopolymer regions of at least 3 base pairs and for which the inserted or deleted base were identical to the homopolymer base were filtered out as homopolymerassociated errors. Indels required a tumour variant allele frequency of $>35 \%$ and all coding indels were manually reviewed using IGV $[30,31]$ in order to be considered high confidence. Variants were annotated with gene feature information and transcript or protein consequences using SnpEff (version 4.2) [32]. Genes of interest, such as Bap1, Cdkn2a and Nf2 were visually inspected for any possible somatic mutation using IGV.

Mutation rate was calculated from the total number of missense, nonsense and silent SNVs plus coding indels divided by the size of the exome library design. Genes significantly mutated at a rate higher than background were identified using Genome MuSIC [33], where a convolution test $p$-value threshold cutoff of $<0.05$ was used to determine significance. Mutations that occurred in all cell lines of the same strain at the same position, but were absent from the matched control were excluded as these were considered to be strain-specific polymorphisms.

\section{Identification of copy number alterations}

Somatic CNVs were identified using coverage information for each tumour exome and matching normal sample calculated by GATK. Normalized and log transformed read count ratios were then used to identify regions of amplification and deletion with ExomeCNV [34]. Segmentation was performed using DNAcopy [35] and segmented data was used as input for the Genetic Identification of Significant Targets in Cancer version 2.0 (GISTIC2.0) program [36] The murine reference genome for GISTIC2.0 was kindly provided by Steven Schumacher (Broad Institute, Cambridge, MA). A q-score of less than 0.1 and a confidence interval of $95 \%$ was used to determine the significance of regions of $\mathrm{CNV}$ across samples. Homozygous deletions of key genes of interest were visually inspected using IGV. To identify whether known tumour suppressor genes, genes in regions of amplification or deletion identified as being significant in the GISTIC2.0 the genes were cross-referenced with the Tumour Suppressor Gene Database 2.0 [37]. 


\section{Identification of altered pathways}

Pathways of interest in MM were identified from the literature. Gene lists for both the human and murine versions of each pathway were extracted from KEGG [38]. All missense, nonsense and frameshift mutations were annotated with corresponding KEGG pathway information for each sample using DAVID Bioinformatics Resources 6.8 [39] and cross referenced with the pathway gene lists. The numbers of genes containing missense, nonsense and frameshift mutations in each pathway of interest was determined. Functional annotation clustering on KEGG pathway annotations using DAVID Bioinformatics Resources 6.8 and WebGestalt [40] was performed for all genes identified as being significantly mutated above background mutation rate and genes located in regions of significant amplification or deletion, with a Benjamini-Hochberg adjusted $p$-value threshold of $<0.05$ from both tools considered significant.

\section{Validation of genetic alterations of interest}

Quantitative real-time polymerase chain reaction (RTPCR) was used to determine mRNA expression. Primers for genes of interest were identified from the literature or designed using Primer-BLAST (NCBI) and purchased from GeneWorks (Thebarton, SA, Australia) (See Additional file 2). Quantitative RT-PCR was performed using SYBR $^{\circ}$ Green Universal PCR Master Mix (ThermoFisher Scientific) for 35 cycles of $95{ }^{\circ} \mathrm{C}$ for 30s, $60{ }^{\circ} \mathrm{C}$ for $30 \mathrm{~s}$ and $72{ }^{\circ} \mathrm{C}$ for $30 \mathrm{~s}$. Differences in gene expression were described as fold-changes between tumour and strain-matched non-tumour sample in order to identify expression changes that may be induced by copy number variation. Sanger sequencing was performed as previously described [25] to confirm the presence of indels.

\section{Results}

Cdkn2a is commonly lost in murine MM tumour cell lines Fifteen murine MM cell lines previously established from ascites generated following intra-peritoneal crocidolite asbestos injection into BALB/c $(n=4)$, CBA $(n=5)$ and C57BL/6 $(n=6)$ mice were assessed for somatic mutations and copy number variation $(\mathrm{CNV})$ aberrations (Table 1) $[4,23,24]$. Overall there were a greater number of copy number deletion events than amplifications. A total of 14 regions were significantly affected by copy number changes, including 12 regions of deletion and 2 regions of gain (See Additional file 3). The most significant region was a homozygous deletion encompassing the tumour suppressor gene Cdkn2a which was evident in all 15 murine MM tumour cell lines ( $\mathrm{q}=5 \times 10^{-17}$ ) (Fig. 1a and $\left.\mathrm{b}\right)$. The breakpoints and length of the region of loss varied between samples, varying from 64.2 to 4025.6 kilobases in length (Table 2). In one sample, AE17, exon 1 of both $C d k n 2 a$ isoforms was retained (NM_001040654.1 and NM_009877.2). Quantitative real-time polymerase chain reaction (PCR) confirmed the absence of $C d k n 2 a$ expression in messenger RNA in all samples (Fig. 1c).

No other significant amplifications or deletions in genes known to be affected in human MM aside from $C d k n 2 a$ were detected in the dataset. However, a number of deleted loci contained known tumour suppressor genes that have been associated with other cancers, as defined by TSGene2.0, such as Epha, Robo1, Arl6, Cadm2, Lipi, Prb1 and Sir1. The number of copy number events did not correlate with time in culture (See Additional file 1).

Copy number aberrations in candidate loci previously implicated in MM were identified in the region of Setd2 in 2 out 15 cell lines and in Lats2 in 3 out of 15 (20\%) cell lines. However, we did not observe reduced mRNA expression of these genes (Fig. 1c) and the regions were not considered to be statistically significantly affected across the samples set through GISTIC2.0 analysis. A region on chromosome 15 encompassing the $M y c$ oncogene was observed to be amplified in 9/15 samples (Fig. 1a and b). Gene expression analysis showed a 150fold increase in expression of Myc in AE17 (Fig. 1c), and a range of 9-to 24-fold increased expression in the other C57BL/6 samples and two CBA samples (AC29, AC31). A homozygous deletion of Trp53 was found in AC29 which was confirmed in the messenger RNA by the absence of product using RT-PCR.

\section{Somatic mutations in murine MM tumour cell lines}

A total of 1286 somatic SNVs and small indels in 1136 genes were detected in the 15 samples. Of these, 899 were missense mutations, 28 were nonsense mutations, 12 were small indels and 347 were silent mutations. On average, there were 84 mutations per sample (range 22 to 438 ) of which $74 \%$ (average 62, range 12 to 327) were protein coding (Fig. 2a; See Additional file 1). Tumours generated in mice of the $\mathrm{BALB} / \mathrm{c}$ background had a higher average number of mutations $(n=225)$ than those from the CBA $(n=33)$ and C57BL/6 $(n=35)$ strains (Fig. 2a; See Additional file 1). The median overall rate of mutation was 0.7 mutations/Mb $(3.9 / \mathrm{Mb}$ for $\mathrm{BALB} / \mathrm{c}, 0.6 / \mathrm{Mb}$ for $\mathrm{CBA}$ and $0.7 / \mathrm{Mb}$ for $\mathrm{C} 57 \mathrm{BL} / 6$ samples). Mutation frequency among BALB/c samples was variable, ranging from 1.4-8.9 mutations/Mb (See Additional file 1).

In general, $\mathrm{C}>\mathrm{T}$ and $\mathrm{G}>\mathrm{A}$ mutations were highly represented in all samples (Fig. 2b). AB13 and AB22 showed marked differences to the other BALB/c samples with an abundance of $\mathrm{C}>\mathrm{G}$ and $\mathrm{A}>\mathrm{T}$ mutations, respectively. For the CBA samples, AC24 showed a higher 


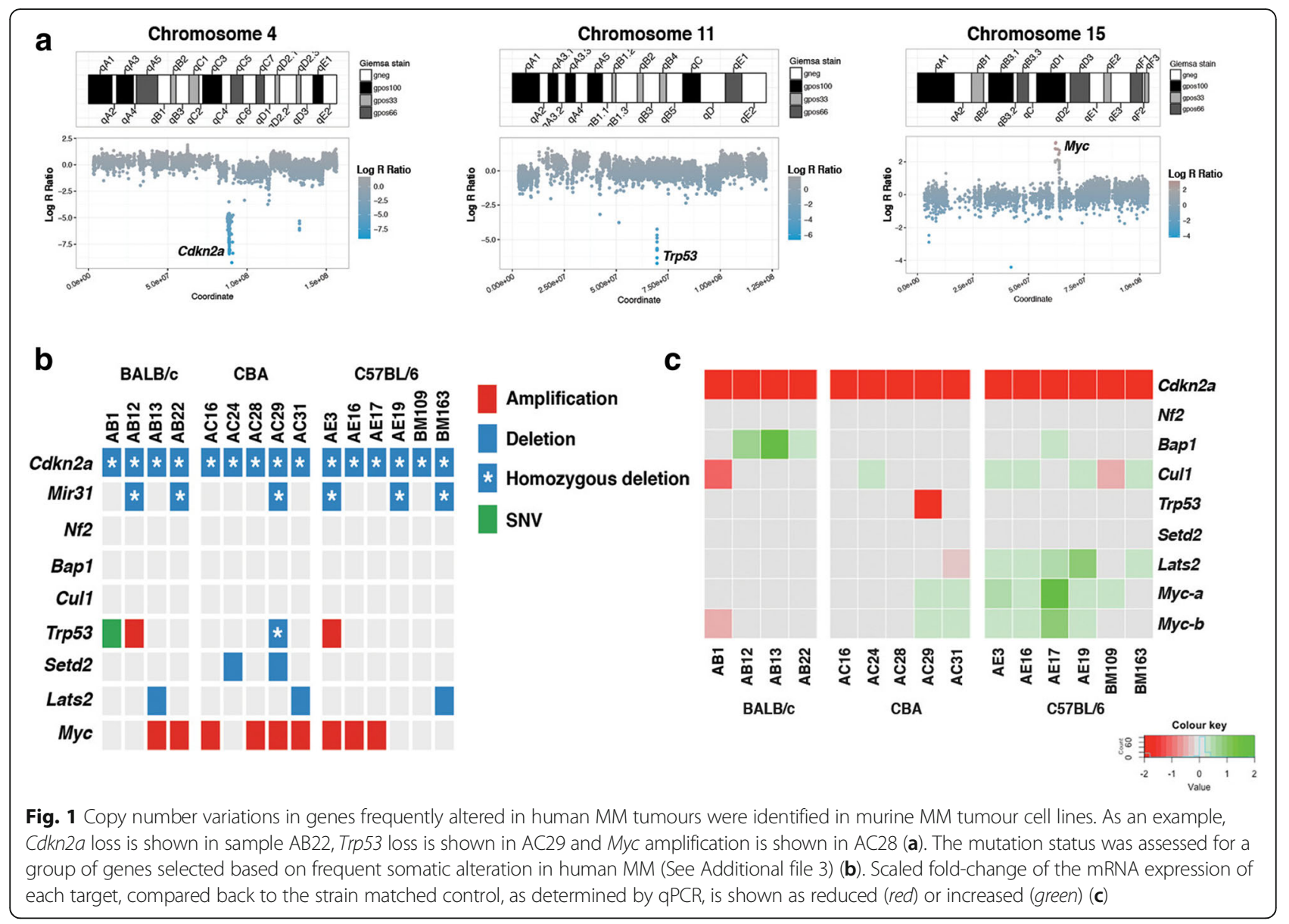

Table 2 Variation in breakpoints of homozygous deletion in region of Cdkn2a

\begin{tabular}{lllll}
\hline Sample & Range (start-end) ${ }^{a}$ & Length (kbp) & $\begin{array}{l}\text { Co-loss of } \\
\text { Mtap }\end{array}$ & $\begin{array}{l}\text { Co-loss of } \\
\text { Mir31 }\end{array}$ \\
\hline AB1 & chr4:88,880,322-89,691,452 & 811.1 & Y & $\mathrm{N}$ \\
AB12 & chr4:88,603,449-91,805,387 & 3201.9 & Y & Y \\
AB13 & chr4:89,217,653-89,443,728 & 226.1 & $\mathrm{~N}$ & $\mathrm{~N}$ \\
AB22 & chr4:88,435,182-90,223,528 & 1788.3 & $\mathrm{Y}$ & $\mathrm{Y}$ \\
AC16 & chr4:89,217,686-89,307,039 & 89.3 & $\mathrm{~N}$ & $\mathrm{~N}$ \\
AC24 & chr4:88,880,305-89,688,653 & 808.3 & $\mathrm{Y}$ & $\mathrm{N}$ \\
AC28 & chr4:89,217,686-89,688,653 & 470.9 & $\mathrm{~N}$ & $\mathrm{~N}$ \\
AC29 & chr4:87,227,653-91,253,295 & 4025.6 & $\mathrm{Y}$ & $\mathrm{Y}$ \\
AC31 & chr4:89,217,686-90,223,513 & 1005.8 & $\mathrm{~N}$ & $\mathrm{~N}$ \\
AE3 & chr4:86,917,707-90,223,511 & 3305.8 & $\mathrm{Y}$ & $\mathrm{Y}$ \\
AE16 & chr4:89,156,673-90,223,511 & 1066.8 & $\mathrm{Y}$ & $\mathrm{N}$ \\
AE17 & chr4:89,217,685-89,281,894 & 64.2 & $\mathrm{~N}$ & $\mathrm{~N}$ \\
AE19 & chr4:87,784,120-90,223,511 & 2439.4 & $\mathrm{Y}$ & $\mathrm{Y}$ \\
BM109 & chr4:89,217,685-89,688,699 & 471.0 & $\mathrm{~N}$ & $\mathrm{~N}$ \\
BM163 & chr4:87,880,239-90,856,876 & 2976.6 & $\mathrm{Y}$ & $\mathrm{Y}$ \\
\hline
\end{tabular}

a Loci reported as per $\mathrm{mm} 10$ coordinates proportion of $\mathrm{G}>\mathrm{T}$ mutations, while the other $\mathrm{CBA}$ samples showed similar proportions of mutation types. Of the C57BL/6 samples, AE19 was dominated by $\mathrm{C}>\mathrm{T}$ at the expense of $\mathrm{G}>\mathrm{A}$ mutations (Fig. 2b).

Fifteen genes were mutated in more than one sample and were found to be significantly mutated above what would be expected as background (Table 3). Several of the genes, such as Mmp8, Dock10, Hoxd3 and Fat3, have previously been reported to show mutation or altered expression in cancer and particularly metastasis [41-45]. Two genes, Cacna2d 2 and $D b c 1$ are candidate tumour suppressors in other cancers [46, 47].

There was no evidence of any mutation in genes most commonly identified as being mutated in human MM, such as Bap1, Nf2 or Lats2. However, a single missense SNV in Trp53 occurred in one BALB/c sample (Fig. 1a). Reduced expression of Bap1 or Nf2 in the messenger RNA was not observed between tumours and normal samples. However two BALB/c samples, AB12 and $A B 13$, showed a 56- and 136-fold increase of Bap1 in mRNA expression, respectively. The genes $R b 1$ and Pten were intact. 

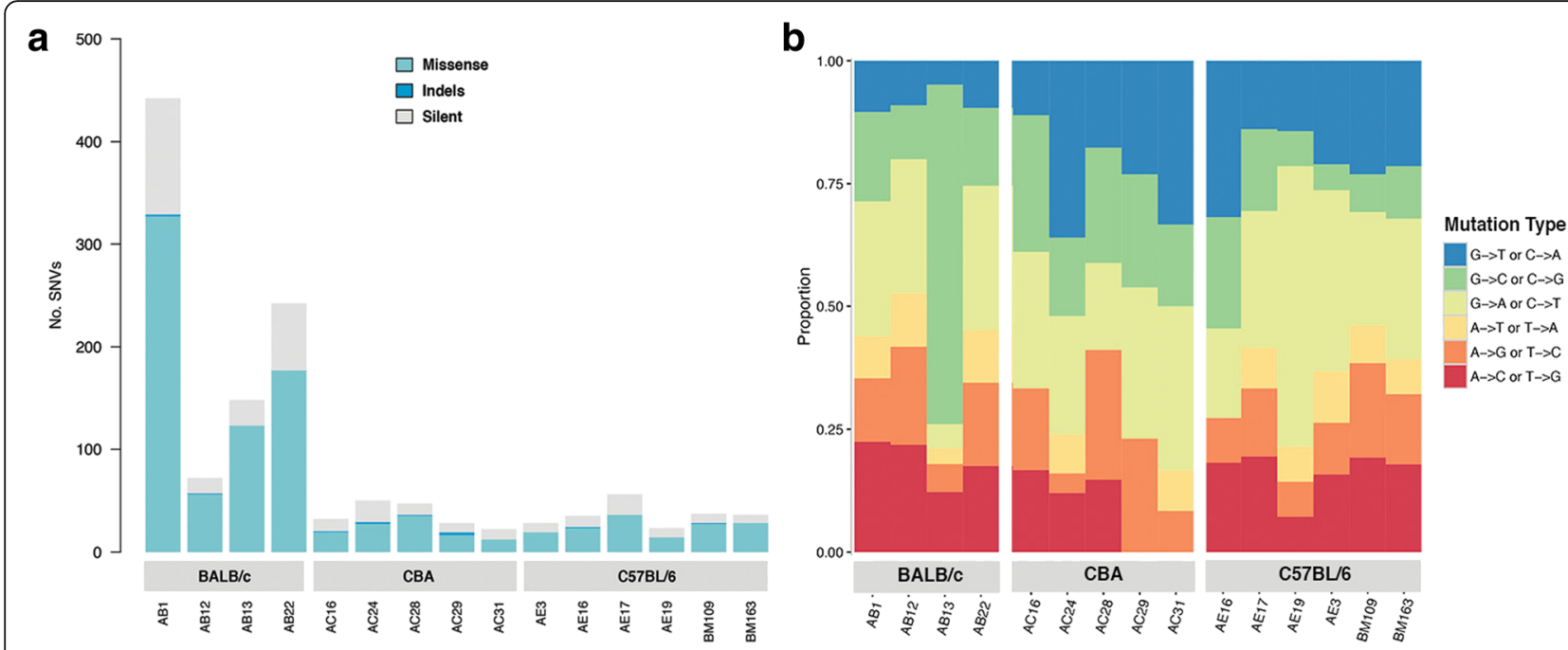

Fig. 2 Exonic somatic mutations in murine MM cell lines showing a breakdown between missense, indels and silent mutations (a) and mutation type (b)

\section{Pathway alterations in murine MM}

Three biological pathways were enriched in the list of genes significantly mutated by somatic mutation or copy number alteration when analysed using WebGestalt and DAVID. Of note the Jak-STAT signaling pathway (WebGestalt adjusted $p$-value $=0.017 ;$ DAVID $p$ value $=0.016)$ were significantly altered (Table 4$)$. Six pathways known to be dysregulated in human MM were affected by missense mutations in the murine MM tumour cell lines: 5/15 samples harbored missense mutations in genes in the Wnt signaling pathway; $3 / 15$ in the Hedgehog signaling pathway; $3 / 15$ in the Notch signaling pathway; $2 / 15$ in the mTOR signaling pathway and $2 / 15$ in the p53 signaling pathway (Fig. 3). All six pathways contained missense mutations in the BALB/c samples. The CBA samples showed mutations in the Wnt, Hedgehog and MAPK signaling pathways, while the C57BL/6 strain contained only one sample carrying a single missense mutation in the MAPK signaling pathway and no mutations in the other five pathways

Table 3 List of genes containing $>1$ amino acid change causing mutation and the significance of the mutation beyond background mutation rate (convoluted $p$-value $<0.05$ )

\begin{tabular}{|c|c|c|c|c|}
\hline Gene (KEGG pathway) & Mutations & Samples affected & No. strains affected & $p$-value \\
\hline Nkd1 (Wnt signalling pathway) & p.P84L|p.V47| & $A B 1 \mid A B 22$ & $1(\mathrm{BALB} / \mathrm{C})$ & $1.34 \mathrm{E}-04$ \\
\hline Mmp8 & p.L7P|p.F303 V & $A B 1 \mid A B 22$ & $1(\mathrm{BALB} / \mathrm{C})$ & $5.38 \mathrm{E}-04$ \\
\hline Nckap5 & p.P1182H, p.L1191 V, p.G768D & $\mathrm{AB} 1|\mathrm{AB} 13| \mathrm{AE} 16$ & $2(\mathrm{BALB} / \mathrm{C} \mid \mathrm{C} 57 \mathrm{BL} / 6)$ & $9.96 \mathrm{E}-04$ \\
\hline Dab1 & p.F203C|p.L502 V & $A B 1$ & $1(\mathrm{BALB} / \mathrm{C})$ & $1.22 \mathrm{E}-03$ \\
\hline Dsc1 & p.K481 N|p.E519D & $A B 1 \mid A B 13$ & $1(\mathrm{BALB} / \mathrm{C})$ & $1.45 \mathrm{E}-03$ \\
\hline Rftn1 & p.V106G|p.W404C & $A B 1 \mid A B 13$ & $1(\mathrm{BALB} / \mathrm{C})$ & $2.43 \mathrm{E}-02$ \\
\hline Ano4 & p.N644 K|p.N406Y & AB22 & $1(\mathrm{BALB} / \mathrm{C})$ & 2.07E-03 \\
\hline Dpp9 & p.T763R|p.Р403Т & AB1 & 1 (BALB/C) & $3.74 \mathrm{E}-03$ \\
\hline Pabpc6 & p.G402R|p.D45Y & $A B 1 \mid A B 22$ & $1(\mathrm{BALB} / \mathrm{C})$ & $3.29 \mathrm{E}-03$ \\
\hline Hoxd3 & p.Q7K|p.V388 M & AE17|AE19 & $1(\mathrm{C} 57 \mathrm{BL} / 6)$ & 4.81E-03 \\
\hline $\mathrm{HC}$ & p.V1332 M|p.|25M & AB22 & $1(\mathrm{BALB} / \mathrm{C})$ & $3.51 \mathrm{E}-03$ \\
\hline Dock10 & p.V1546G|p.A2076V & $\mathrm{AB} 1 \mid \mathrm{AE} 17$ & $2(\mathrm{BALB} / \mathrm{C} \mid \mathrm{C} 57 \mathrm{BL} / 6)$ & $3.28 \mathrm{E}-03$ \\
\hline Cacna2d2 (MAPK signalling pathway) & p.K857 M|p.R575Q & AB1|AC28 & $2(\mathrm{BALB} / \mathrm{C} \mid \mathrm{CBA})$ & 3.67E-03 \\
\hline Neurl4 & p.V1163||p.T832 K & AC29|BM163 & $2(\mathrm{CBA} \mid \mathrm{C} 57 \mathrm{BL} / 6)$ & $6.50 \mathrm{E}-03$ \\
\hline Fat3 & p.D2918G|p.D1300E & $\mathrm{AB} 1$ & $1(\mathrm{BALB} / \mathrm{C})$ & $3.61 \mathrm{E}-02$ \\
\hline
\end{tabular}


Table 4 KEGG pathways enriched from list of significantly mutated genes and significantly amplified or deleted regions $(n=804)$

\begin{tabular}{llll}
\hline KEGG pathway & No. mutated genes & BH adjusted p-value (WebGestalt) & BH adjusted $p$-value (DAVID) \\
\hline Regulation of autophagy & 7 & $1.5 \times 10^{-3}$ & $5.2 \times 10^{-4}$ \\
RIG-I-like receptor signaling pathway & 9 & $2.4 \times 10^{-3}$ & $1.1 \times 10^{-2}$ \\
Jak-STAT signaling pathway & 12 & $1.7 \times 10^{-2}$ & $1.6 \times 10^{-2}$ \\
\hline
\end{tabular}

(Fig. 3). There were no mutations in the Ras signaling pathway, however Lats2, which initiates cell apoptosis through the Hippo pathway, contained copy number deletions in 3/15 samples.

\section{Discussion}

The asbestos-induced wild-type murine model of MM has the power to provide invaluable insights into the pathology of human MM, provided that the tumours are similar on a molecular basis between the species. Through whole exome sequencing of tumour cell lines developed from mesotheliomas from three murine strains, we have shown that the $C d k n 2 a$ gene is consistently lost in the asbestos-induced wild-type murine model of MM. This parallels the situation in more than $70 \%$ of human MM tumours [12]. Previously, in a genetically engineered MM mouse model, heterozygous for $N f 2^{+/-}$, a high rate of homozygous deletion of $C d k n 2 a$ was observed in MM tumours induced by asbestos exposure [19]. In the human context $C D K N 2 A$ loss, deletion of NF2 and/or mutation of $B A P 1$ has been reported in up to $80 \%$ of clinical cases [8]. In our model we did not find mutations, copy number deletions or reduced mRNA expression of Bap1, however, loss of BAP1 protein expression has been reported in up to $25 \%$ of cases where no genetic mutations were detected [9]. Dysregulation of the Hippo pathway is often reported in human $\mathrm{MM}$, typically through mutations or copy number aberrations of NF2, YAP and/or LATS2 [14, 48]. No mutations in Lats2 were observed in this study, however $20 \%$ of samples in this study contained a copy number deletion of Lats2. With post-transcriptional modification potentially accounting for loss of these tumour suppressors in the mouse asbestos induced MM model it is not possible to conclusively say that $C d k n 2 a$ deletion is an independent driver of MM. However it seems that homozygous loss of the $C d k n 2 a$ gene is sufficient for the development of asbestos-induced MM in the absence of other common MM driver mutations at the genetic and transcriptomic level in this model.

Though most human MMs develop in the pleura, the murine model used in this study developed in the peritoneum. Despite this difference, the developmental characteristics of murine MM faithfully mimic human MM [4]. Pleural and peritoneal MM are different in terms of prognosis and treatment options, however the molecular features of peritoneal MM are not as well characterised as pleural MM, given the rarity of the diagnosis. It is not uncommon to have secondary pleural invasion from peritoneal MM and vice versa, and in these cases the distinction between the two types is clinically difficult [49]. The histological feature of pleural and peritoneal MM are generally identical, and on this basis, the molecular characterisation of murine MM in this study will provide the necessary basis for future studies seeking to target specific molecular pathways for therapy.

The asbestos-exposed wild-type murine model of MM displayed a high proportion of chromosomal loss

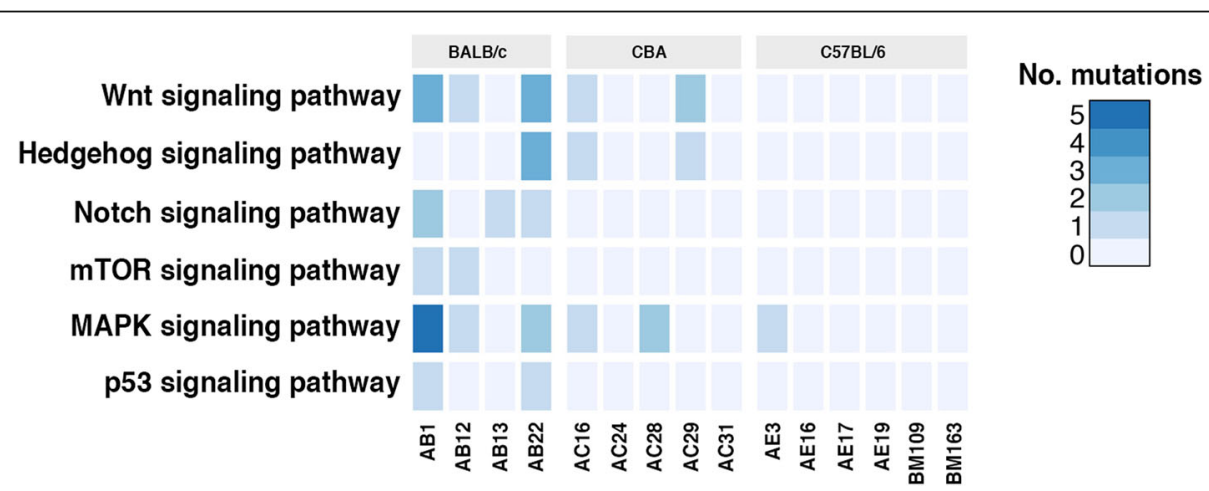

Fig. 3 The number of missense mutations affecting pathways commonly known to be disregulated in human MM tumours. The following number of genes contained missense mutations for each pathway: 10 (Wnt signaling pathway), 5 (Hedgehog signaling pathway), 2 (Notch signaling pathway), 2 (mTOR signaling pathway), 11 (MAPK signaling pathway) and 2 (p53 signaling pathway) 
compared to gain, which is a characteristic feature of human MM. Human MM tumours display recurrent losses in 1p21-p22, 9p21-p22, 3p21, 6q15-q21, 17p13.1 and 22q12.2 [50, 51]. Taking into account the locations of $B A P 1, N F 2$ and $C D K N 2 A$ in the human genome, the only orthologous region containing $\mathrm{CNV}$ aberration in this model was the region containing $C d k n 2 a$ (4qC4). Previously we have shown using our MexTAg transgenic mouse model [24] that the presence of exogenous SV40 large $\mathrm{T}$ antigen (Tag) performs a similar molecular role as Cdkn2a loss [21] In both the wild type and MexTAg model, asbestos-induced MM was not associated with reduced expression of other genes commonly downregulated in human MM [21].

To date no common oncogenic driver has been identified in MM, although amplification of the C-MYC locus in $\mathrm{MM}$ has been reported to occur in MM human tumour cell lines and may contribute to a malignant phenotype [52]. In the current study, increased expression of c-Myc was observed, however no other recognized oncogenes were identified in significantly amplified regions across the samples. It is important to acknowledge that, despite these particular CNVs being previously reported in human MM, the selection pressure of culture conditions may account for some CNV in this study. Notably, however, there was no correlation between the number of CNVs and time in culture in this study.

Carcinogen-induced cancers such as melanoma and lung cancer typically show a high rate of mutation when compared to spontaneous cancers [6-8]. Given that $\mathrm{MM}$ is also a carcinogen induced tumour, the average somatic mutation rate in human MM is lower than expected $[7,8]$. Here we show that the CBA and C57BL/6 strains of the wild-type murine model of MM display a comparable mutation rate to human MM. Interestingly, the BALB/c strain showed a wider range of mutation rates between the samples. Previously, germline mutations in a key DNA damage sensor gene, $\operatorname{Prkdc}$, have been reported in some colonies of BALB/c mice [53]. These mutations were also present in the $\mathrm{BALB} / \mathrm{c}$ mice used to generate the cell lines used in this study, possibly resulting in the current and previously reported genomic instability of tumour cell lines from this strain [5] and may account for the higher rate of spontaneous mutation when exposed to asbestos.

Patterns of mutations can be used to identify mutational signatures which represent the underlying process of DNA damage, (for example tobacco smoking induces a mutational pattern that exhibits transcriptional strand bias for $\mathrm{C}>\mathrm{A}$ mutations and CC > AA dinucleotide substitutions) [6]. To date no asbestos-related mutation signature has been identified.
The current exome sequencing study was underpowered to perform a full mutational signature analysis but did show a high rate of $\mathrm{C}>\mathrm{T}$ and $\mathrm{G}>\mathrm{A}$ mutations, consistent with previous studies in humans $[7,8]$. Whole genome sequencing of a larger cohort of samples will be required to fully answer this question in both humans and mice.

Given the genetically independent nature of $\mathrm{MM}$ tumours and the low likelihood of the same gene being mutated at different positions in more than one sample, the presence of different mutations in $N k d 1$ in two BALB/c samples and Cacna2d2 in samples from two different strains is informative. The mutation of $N k d 1$ may implicate the role of Wnt signaling pathway regulation in the BALB/c strain. Genes involved in the Jak-STAT and MAPK signaling pathways were also altered in the murine model. These Wnt, Jak-STAT and MAPK pathways have been identified as being altered in many cancers, including MM. In humans, CACNA2D2 is located $1.9 \mathrm{Mb}$ upstream from $B A P 1$ in the region $3 \mathrm{p} 21$. However, in the mouse genome, the orthologs reside on different chromosomes. The importance of this gene in $\mathrm{MM}$ in undetermined, though it has been linked to tumorigenesis in prostate cancer [46] and loss of expression has been demonstrated in non-small cell lung cancer [54].

\section{Conclusion}

Our study is the first to molecularly characterise three extensively utilized strains of a wild-type model of asbestos-induced MM and catalogue the somatic mutations and copy number variations they contain. Previous studies have reported on small numbers of wild-type murine MM tumours within larger studies focusing on transgenic models $[5,19,20,22,24]$. We confirm that asbestos exposure in wild-type mice causes MM tumour development through homozygous loss of $C d k n 2 a$ leading to loss of expression of p16 and that this is an initiating event of MM tumorigenesis, a loss that is commonly identified in over $70 \%$ of human MM tumours. We find that wild-type asbestos-induced murine MM harbors a similar mutation rate to human MM and that copy number deletions dominate the mutational landscape, particularly within similar pathways as human MM. This study provides a foundation for further research into the wild-type murine MM model at the translational level. Our findings provide support for the use of a wild-type murine model of asbestos-induced malignant mesothelioma as an invaluable tool for the study of the molecular basis of human MM, as well as provide the means to study early events in MM development post asbestos exposure, the search for novel therapies based on identification of druggable molecular targets, carcinogen signature evaluation and biomarker studies. 


\section{Additional files}

Additional file 1: Table S1. Sequencing metrics and summary of somatic mutations and copy number variations. (DOCX $111 \mathrm{~kb}$ )

Additional file 2: Table S2. Genes of interest previously reported in MM and primer sequences used to detect expression of the targets in murine messenger RNA. (DOCX $85 \mathrm{~kb}$ )

Additional file 3: Table S3. Copy number variations determined as significant across all 15 tumour cell line samples (q-value threshold <0.1). (DOCX $141 \mathrm{~kb})$

\section{Abbreviations}

aCGH: Array comparative genomic hybridisation; Bap1: BRCA1 associated protein 1; Cdkn2a: Cyclin dependent kinase inhibitor 2A; CIGAR: Concise idiosyncratic gapped alignment report; CNV: Copy number variation; DNA: Deoxyribonucleic acid; GATK: Genome Analysis Toolkit; GISTIC: Genetic identification of significant targets in cancer; IGV: Integrative Genomics Viewer; Indel: Short insertion or deletion; KEGG: Kyoto Encyclopedia of Genes and Genomes; Mb: Million bases; MD: Match/mismatch; MM: Mesothelioma; mRNA: Messenger RNA; Nf2: Neurofibromin 2; RNA: Ribonucleic acid; RTPCR: Reverse transcription polymerase chain reaction; SNV: Single nucleotide variation; Trp53: Tumour protein 53; UV: Ultraviolet; -x: -fold coverage

\section{Acknowledgements}

Not applicable.

\section{Funding}

This work was supported in part by the Housing Industry of Australia Charitable Foundation, the Insurance Commission of Western Australia and the Australian National Health and Medical Research Council (NHMRC) (APP1089404). SS receives a scholarship from the Ross Divett Foundation. NW is supported by a NHMRC Career Development Fellowship (1112113). BR is supported by a NHMRC Practitioner fellowship (1108638). The funding agencies played no part in study design, data analysis, interpretation of data or manuscript preparation.

\section{Availability of data and materials}

The raw data generated during and/or analysed during the current study are available in the NCBI Sequence Read Archive (SRA) repository under the project ID PRJNA342560, (http://www.ncbi.nlm.nih.gov/bioproject/ PRJNA342560)

\section{Authors' contributions}

Experiment design: SS, JC, BR. Data generation: SS, RA. Sequence data analysis: SS, AMP, SK, JVP, NW, JC, ID, BR. Manuscript preparation: SS, JC, ID, AMP, NW, RA, ID, RH, BR. All authors have read and approved this manuscript.

\section{Competing interests}

The authors declare no competing interests.

\section{Consent for publication}

Not applicable.

\section{Ethics approval and consent to participate}

Control DNA and RNA used in this study were obtained from the Animal Resource Centre (Murdoch, WA, Australia) in accordance with protocols approved by the University of Western Australia Animal Ethics Committee. This study complies with the Animal Welfare Act (2002) (http:// www.austlii.edu.au/au/legis/wa/consol_act/awa2002128/) and the Australian code for the care and use of animals for scientific purposes (8th Edition, 2013) (https://www.nhmrc.gov.au/guidelines-publications/ea28).

\section{Publisher's Note}

Springer Nature remains neutral with regard to jurisdictional claims in published maps and institutional affiliations.

\section{Author details}

'National Centre for Asbestos Related Disease, School of Medicine and Pharmacology, QEIl Medical Centre, University of Western Australia, QQ Block,
6 Verdun Street, Nedlands, WA 6009, Australia. ${ }^{2}$ QIMR Berghofer Medical Research Institute, Brisbane, Brisbane, QLD 4006, Australia. ${ }^{3}$ School of Pathology and Laboratory Medicine, University of Western Australia, Nedlands, WA 6009, Australia. ${ }^{4}$ Pathwest Laboratory Medicine, Western Australia, QEIl Medical Centre, Nedlands, WA 6009, Australia. ${ }^{5}$ Michael Smith Genome Sciences Centre, BC Cancer Agency, Vancouver, BC, Canada. ${ }^{6}$ Department of Respiratory Medicine, Sir Charles Gairdner Hospital, Nedlands, WA 6009, Australia.

Received: 3 November 2016 Accepted: 23 May 2017

Published online: 02 June 2017

\section{References}

1. Robinson BW, Lake RA. Advances in malignant mesothelioma. N Engl J med. 2005:353:1591-603.

2. Robinson BW, Musk AW, Lake RA. Malignant mesothelioma. Lancet. 2005:366:397-408.

3. Wagner JC, Sleggs CA, Marchand P. Diffuse pleural mesothelioma and asbestos exposure in the north Western Cape Province. Br J Ind med. 1960;17:260-71.

4. Davis MR, Manning LS, Whitaker D, Garlepp MJ, Robinson BW. Establishment of a murine model of malignant mesothelioma. Int J Cancer. 1992;52:881-6.

5. Mezzapelle R, Rrapaj E, Gatti E, Ceriotti C, Marchis FD, Preti A, et al. Human malignant mesothelioma is recapitulated in immunocompetent BALB/C mice injected with murine AB cells. Sci rep. 2016;6:22850.

6. Alexandrov LB, Nik-Zainal S, Wedge DC, Aparicio SA, Behjati S, Biankin AV, et al. Signatures of mutational processes in human cancer. Nature. 2013; 500:415-21.

7. Bueno R, Stawiski EW, Goldstein LD, Durinck S, De Rienzo A, Modrusan Z, et al. Comprehensive genomic analysis of malignant pleural mesothelioma identifies recurrent mutations, gene fusions and splicing alterations. Nat Genet. 2016;48:407-16

8. Guo G, Chmielecki J, Goparaju C, Heguy A, Dolgalev I, Carbone M, et al. Whole-exome sequencing reveals frequent genetic alterations in BAP1, NF2, CDKN2A, and CUL1 in malignant pleural mesothelioma. Cancer Res. 2015;75:264-9.

9. Bott M, Brevet M, Taylor BS, Shimizu S, Ito T, Wang $L$, et al. The nuclear deubiquitinase BAP1 is commonly inactivated by somatic mutations and 3p21.1 losses in malignant pleural mesothelioma. Nat Genet. 2011;43:668-72.

10. Deguen B, Goutebroze L, Giovannini M, Boisson C, van der Neut R, Jaurand $M C$, et al. Heterogeneity of mesothelioma cell lines as defined by altered genomic structure and expression of the NF2 gene. Int J Cancer. 1998;77:554-60.

11. Sekido Y, Pass HI, Bader S, Mew DJ, Christman MF, Gazdar AF, et al. Neurofibromatosis type 2 (NF2) gene is somatically mutated in mesothelioma but not in lung cancer. Cancer Res. 1995:55:1227-31.

12. Illei PB, Rusch WW, Zakowski MF, Ladanyi M. Homozygous deletion of CDKN2A and codeletion of the methylthioadenosine phosphorylase gene in the majority of pleural mesotheliomas. Clin Cancer Res. 2003;9:2108-13.

13. Bianchi AB, Mitsunaga SI, Cheng JQ, Klein WM, Jhanwar SC, Seizinger B, et al. High frequency of inactivating mutations in the neurofibromatosis type 2 gene (NF2) in primary malignant mesotheliomas. Proc Natl Acad Sci U S A. 1995:92:10854-8.

14. Murakami H, Mizuno T, Taniguchi T, Fujii M, Ishiguro F, Fukui T, et al. LATS2 is a tumor suppressor gene of malignant mesothelioma. Cancer Res. $2011 ; 71: 873-83$

15. Fox SA, Richards AK, Kusumah I, Perumal V, Bolitho EM, Mutsaers SE, et al. Expression profile and function of Wnt signaling mechanisms in malignant mesothelioma cells. Biochem Biophys res Commun. 2013:440:82-7.

16. Shi Y, Moura U, Opitz I, Soltermann A, Rehrauer H, Thies S, et al. Role of hedgehog signaling in malignant pleural mesothelioma. Clin Cancer Res. 2012;18:4646-56.

17. Robinson C, Solin JN, Lee YCG, Lake RA, Lesterhuis WJ. Mouse models of mesothelioma: strengths, limitations and clinical translation. Lung Cancer Management. 2014;3:397-410.

18. Dey A, Seshasayee D, Noubade R, French DM, Liu J, Chaurushiya MS, et al. Loss of the tumor suppressor BAP1 causes myeloid transformation. Science. 2012;337:1541-6.

19. Altomare DA, Vaslet CA, Skele KL, De Rienzo A, Devarajan K, Jhanwar SC, et al. A mouse model recapitulating molecular features of human mesothelioma. Cancer Res. 2005;65:8090-5. 
20. Altomare DA, Menges CW, Xu J, Pei J, Zhang L, Tadevosyan A, et al. Losses of both products of the Cdkn2a/Arf locus contribute to asbestos-induced mesothelioma development and cooperate to accelerate tumorigenesis. PLoS One. 2011;6:e18828.

21. Robinson C, Dick IM, Wise MJ, Holloway A, Diyagama D, Robinson BW, et al. Consistent gene expression profiles in MexTAg transgenic mouse and wild type mouse asbestos-induced mesothelioma. BMC Cancer. 2015;15:983.

22. Jean D, Thomas E, Manie E, Renier A, de Reynies A, Lecomte C, et al. Syntenic relationships between genomic profiles of fiber-induced murine and human malignant mesothelioma. Am J Pathol. 2011;178:881-94.

23. Jackaman C, Bundell CS, Kinnear BF, Smith AM, Filion P, van Hagen D, et al. IL-2 intratumoral immunotherapy enhances CD8+ T cells that mediate destruction of tumor cells and tumor-associated vasculature: a novel mechanism for IL-2. J Immunol. 2003;171:5051-63.

24. Robinson C, van Bruggen I, Segal A, Dunham M, Sherwood A, Koentgen F, et al. A novel SV40 TAg transgenic model of asbestos-induced mesothelioma: malignant transformation is dose dependent. Cancer Res. 2006;66:10786-94.

25. Creaney J, Ma S, Sneddon SA, Tourigny MR, Dick IM, Leon JS, et al. Strong spontaneous tumor neoantigen responses induced by a natural human carcinogen. Onco Immunology. 2015;4:e1011492

26. Martin M: Cutadapt removes adapter sequences from high-throughput sequencing reads. 2011 2011, 17

27. Li H, Handsaker B, Wysoker A, Fennell T, Ruan J, Homer N, et al. The Sequence alignment/map format and SAMtools. Bioinformatics. 2009;25:2078-9.

28. Kassahn KS, Holmes O, Nones K, Patch AM, Miller DK, Christ AN, et al. Somatic point mutation calling in low cellularity tumors. PLoS One. 2013;8:e74380.

29. McKenna A, Hanna M, Banks E, Sivachenko A, Cibulskis K, Kernytsky A, et al. The genome analysis Toolkit: a MapReduce framework for analyzing nextgeneration DNA sequencing data. Genome Res. 2010;20:1297-303.

30. Thorvaldsdottir H, Robinson JT, Mesirov JP. Integrative genomics viewer (IGV): high-performance genomics data visualization and exploration. Brief Bioinform. 2013;14:178-92.

31. Robinson JT, Thorvaldsdottir H, Winckler W, Guttman M, Lander ES, Getz G, et al. Integrative genomics viewer. Nat Biotechnol. 2011;29:24-6.

32. Cingolani P, Platts A, Wang le L, Coon M, Nguyen T, Wang L, Land SJ, Lu X, Ruden DM: A program for annotating and predicting the effects of single nucleotide polymorphisms, SnpEff: SNPs in the genome of Drosophila melanogaster strain W1118; iso-2; iso-3. Fly (Austin). 2012;6:80-92.

33. Dees ND, Zhang Q, Kandoth C, Wendl MC, Schierding W, Koboldt DC, et al. MuSiC: identifying mutational significance in cancer genomes. Genome res. 2012;22:1589-98

34. Sathirapongsasuti JF, Lee H, Horst BA, Brunner G, Cochran AJ, Binder S, et al. Exome sequencing-based copy-number variation and loss of heterozygosity detection: ExomeCNV. Bioinformatics. 2011;27:2648-54.

35. Olshen $A B$, Venkatraman ES, Lucito R, Wigler M. Circular binary segmentation for the analysis of array-based DNA copy number data. Biostatistics. 2004;5:557-72

36. Mermel CH, Schumacher SE, Hill B, Meyerson ML, Beroukhim R, Getz G. GISTIC2.0 facilitates sensitive and confident localization of the targets of focal somatic copy-number alteration in human cancers. Genome Biol. 2011;12:R41.

37. Zhao M, Kim P, Mitra R, Zhao J, Zhao Z. TSGene 2.0: an updated literaturebased knowledgebase for tumor suppressor genes. Nucleic Acids res. 2016:44:D1023-31

38. Kanehisa M, Sato Y, Kawashima M, Furumichi M, Tanabe M. KEGG as a reference resource for gene and protein annotation. Nucleic Acids res. 2016;44:D457-62.

39. Huang da W, Sherman BT, Lempicki RA. Systematic and integrative analysis of large gene lists using DAVID bioinformatics resources. Nat Protoc 2009;4:44-57.

40. Wang J, Duncan D, Shi Z, Zhang B. WEB-based GEne SeT AnaLysis Toolkit (WebGestalt): update 2013. Nucleic Acids res. 2013;41:W77-83.

41. De Craene B, Berx G. Regulatory networks defining EMT during cancer initiation and progression. Nat rev Cancer. 2013;13:97-110.

42. Westcott JM, Prechtl AM, Maine EA, Dang TT, Esparza MA, Sun H, et al. An epigenetically distinct breast cancer cell subpopulation promotes collective invasion. J Clin Invest. 2015:125:1927-43.
43. Shaoqiang C, Yue Z, Yang L, Hong Z, Lina Z, Da P, et al. Expression of HOXD3 correlates with shorter survival in patients with invasive breast cancer. Clin Exp Metastasis. 2013;30:155-63.

44. Longacre M, Snyder NA, Housman G, Leary M, Lapinska K, Heerboth S, et al. A comparative analysis of genetic and epigenetic events of breast and ovarian cancer related to tumorigenesis. Int J Mol Sci. 2016;17:759-75.

45. Gutiérrez-Fernández A, Fueyo A, Folgueras AR, Garabaya C, Pennington CJ, Pilgrim S, et al. Matrix metalloproteinase-8 functions as a metastasis suppressor through modulation of tumor cell adhesion and invasion. Cancer Res. 2008;68:2755-63.

46. Warnier M, Roudbaraki M, Derouiche S, Delcourt P, Bokhobza A, Prevarskaya $N$, et al. CACNA2D2 promotes tumorigenesis by stimulating cell proliferation and angiogenesis. Oncogene. 2015;34:5383-94.

47. Hamaguchi M, Meth JL, von Klitzing C, Wei W, Esposito D, Rodgers L, et al. $\mathrm{DBC2}$, a candidate for a tumor suppressor gene involved in breast cancer. Proc Natl Acad Sci U S A. 2002;99:13647-52.

48. Mizuno T, Murakami H, Fujii M, Ishiguro F, Tanaka I, Kondo Y, et al. YAP induces malignant mesothelioma cell proliferation by upregulating transcription of cell cycle-promoting genes. Oncogene. 2012;31:5117-22.

49. Trupiano JK, Geisinger KR, Willingham MC, Manders P, Zbieranski N, Case D, et al. Diffuse malignant mesothelioma of the peritoneum and pleura, analysis of markers. Mod Pathol. 2004;17:476-81.

50. Taguchi T, Jhanwar SC, Siegfried JM, Keller SM, Testa JR. Recurrent deletions of specific chromosomal sites in 1p, 3p, 6q, and $9 p$ in human malignant mesothelioma. Cancer Res. 1993;53:4349-55.

51. Ivanov SV, Miller J, Lucito R, Tang C, Ivanova AV, Pei J, et al. Genomic events associated with progression of pleural malignant mesothelioma. Int J Cancer. 2009;124:589-99.

52. Riquelme E, Suraokar MB, Rodriguez J, Mino B, Lin HY, Rice DC, et al. Frequent coamplification and cooperation between C-MYC and PVT1 oncogenes promote malignant pleural mesothelioma. J Thorac Oncol. 2014;9:998-1007.

53. Barber R, Plumb MA, Boulton E, Roux I, Dubrova YE. Elevated mutation rates in the germ line of first- and second-generation offspring of irradiated male mice. Proc Natl Acad Sci U S A. 2002;99:6877-82.

54. Xinarianos G, Panutsopulos D, Fielding P, Gosney JR, Spandidos DA, Liloglou T, Field JK: Loss of CACNA2D2 expression in non-small cell lung cancer. Cancer Res. 2006;66:986-86.

\section{Submit your next manuscript to BioMed Central and we will help you at every step:}

- We accept pre-submission inquiries

- Our selector tool helps you to find the most relevant journal

- We provide round the clock customer support

- Convenient online submission

- Thorough peer review

- Inclusion in PubMed and all major indexing services

- Maximum visibility for your research

Submit your manuscript at www.biomedcentral.com/submit
) Biomed Central 